\title{
Use and understanding of current UK nutrition label information
}

\author{
S.G. Moore ${ }^{1}$ J. Donnelly ${ }^{1}$, S. Jones ${ }^{1}$ and J.E Cade ${ }^{2}$ \\ ${ }^{1}$ Leeds Trinity University School of Social and Health Sciences, Leeds, LS18 5HD, UK and ${ }^{2}$ School of Food Science \\ and Nutrition, University of Leeds, Leeds, LS2 9JT, UK.
}

Nutrition information has been available in the UK for decades but became mandatory from 2014 on food labels and on webpages for products sold online ${ }^{(1)}$. Prior to this change, review evidence suggests more than $50 \%$ of consumers report using nutrition information (NI) with variations by subgroup (e.g. age and sex $)^{(2)}$. Globally, use of NI has been linked with healthier diets ${ }^{(2)}$ and consumer's personal motivations ${ }^{(3)}$. Following the implementation of the new UK NI, the first aim of this research was to evaluate consumer use of this information, including when online grocery shopping. Understanding of NI is linked to label use ${ }^{(3)}$ yet there is currently no literature evaluating consumer understanding of the current UK mandatory Back-of-Pack (BoP) nutrition labelling. Previous research into consumer understanding of NI has focussed on the impact of various formats of voluntary Front-of-Pack (FoP) labels and consumers' level of nutrition knowledge ${ }^{(3)}$. Research into consumer understanding of pre-2014 BoP NI in the UK and US has identified specific difficulties with interpreting percentages and servings sizes ${ }^{(2)}$. Since the new UK NI uses different terminology alongside serving sizes and percentage data, this research also aimed to evaluate consumers' understanding.

In 2015 an online survey was devised to assess use and objective understanding of the new NI. The survey was completed by 181 adults (mean age 58.6, SD 7.7yrs, $90 \%$ white British, $73 \%$ female, and $65 \%$ university educated). The majority ( $71 \%$ ) reported frequently reading NI the first time they purchased a product. Those who reported frequent reading in 'general shopping' (51 \%) or stated NI affected their purchase choices $(51 \%)$, were significantly more likely to report higher personal involvement with NI than infrequent readers $(\mathrm{p}<.001)$. Respondents who perceived NI as 'easy' to understand were also significantly more likely to report that this information frequently affected their purchase choices $(p=.041)$. Respondents included 70 online shoppers, of which only $19 \%$ reported either frequent reading of NI in online shopping or that this information frequently affected their online purchase choices.

An objective NI comprehension test was also completed by all survey respondents. This used six survey questions concerning locating or defining label data and "Reference Intake" (RI) terminology (formerly Guideline Daily Amounts, GDA). Respondents' mean score was $(65 \%$ SD $25 \%)$. There were no statistically significant differences in overall comprehension scores between frequent and infrequent NI label users. Increasing comprehension score was found to be associated with level of nutrition knowledge ( $\mathrm{p}<\cdot 001)$. Quiz questions with the lowest number of correct responses concerned the meaning of the terms 'Reference Intakes' (RI) (54\% correct) and 'RI for fat' ( $44 \%$ correct). In addition, only $59 \%$ of shoppers were able to use a label to correctly locate the "percentage of your reference intake for saturates provided by a serving".

In conclusion, levels of use and impact of NI on 'labels' are higher than for nutrition information displayed during online shopping. Results suggest limited comprehension of BoP nutrient data and terminology in this sample. However, there is no evidence that those with higher comprehension scores are more likely to read or use them to affect their purchase choices. The findings warrant further investigation of other population groups and development of strategies to improve adult comprehension of mandatory NI.

1. Butriss J. (2018) Proc Nutr Soc 1,1-10.

2. Campos S, Doxey J \& Hammond D. (2011) Public Health Nutr 14(8), 1496-1506.

3. Grunert KG, Wills JM \& Fenandez-Clemin L (2010) Appetite 55, 166-189. 